\title{
Surfactant induced stabilization of nano liquid crystalline (dodecane-phytantriol) droplet
}

\author{
S. Abbas ${ }^{1 a}$, Debasish Saha, Sugam Kumar ${ }^{1}$, V. K. Aswal ${ }^{1}$ and J. Kohlbrecher ${ }^{2}$ \\ ${ }^{1}$ Solid State Physics Division, Bhabha Atomic Research Centre, Mumbai 400 085, India \\ ${ }^{2}$ Laboratory for Neutron Scattering, Paul ScherrerInstitut, CH-5232 PSI Villigen, Switzerland \\ ${ }^{a)}$ Corresponding author:abbas@barc.gov.in
}

\begin{abstract}
The study of formation and stabilization of dodecane -phytantriol (DPT) microemulsions using ionic and nonionic surfactants are investigated. Small Angle Neutron Scattering (SANS) and Dynamic Light Scattering (DLS) techniques have been employed to study the resulting structures of the micro emulsion droplets. We show the formation of stable microemulsion droplets with absence of lyotropic liquid crystalline phase on addition of nonionic surfactant C12E10. The oil to surfactant ratio plays the crucial role in formation of stable droplet and its size. The dense presence of C12E10 molecules between microemulsion droplets protect them from coalescence while less number of C12E10 between the surface of droplets easily triggers the coalescence process. The interaction with both anionic (SDS) as well as cationic (DTAB) surfactants with DPT phase leads to formation of microemulsion droplets with lyotropic liquid crystalline phase.
\end{abstract}

\section{INTRODUCTION}

Since its discovery [1], microemulsions have attained great significance both in basic research as well as in industrial applications. The microemulsions find numerous usages in chemical and biological fields [2].This is largely attributed to their unique properties such as large interfacial area, small interfacial tension, thermodynamic stability etc. [3]. The emerging trend in using such microemulsion lies in droplet functionalisation and creating the smart functional materials [4-6]. Reverse micelles "Water in oil microemulsion" finds applications in synthetic chemistry,synthesis of biologically important systems etc. [ ]. Recently the use of lyotropic liquidcrystalline (LC) structures as the dispersed phase has attracted a lot of attention due to its numerous applications in pharmaceutics, food, cosmetics etc. [ ]. The stabilization dodecane-phytantriol(DPT) system has been studied using colloids, $\mathrm{pH}$ and surfactant [ ].

The interdroplet interaction in microemulsions can be tuned by changing the number of surfactants cover the droplets. More number of surfactant molecules on the surface stabilized the droplets against coalescence through the electrostatic interaction (ionic surfactant) or hydrophobic interaction (nonionic surfactant). In order to address this issue we have studied the DPTmicromeuslion system and its stabilization and size control using nonionic surfactant (C12E10) at different concentrations. Microemulsiondropelts were stabilized well upto the DPT and surfactant ratio 1:5. In further increasing DPT content, coalescence of droplets was observed. To compare the stabilization ability of different surfactants, an anionic sodium dodecyl sulfate (SDS) and a cationic Dodecyltrimethylammonium bromide (DTAB) surfactants with DPT were further examined. The scattering techniques (SANS and DLS) have been used to study the structure and interaction of the droplets as afunction of surfactant to DPT phase. 


\section{EXPERIMENTAL}

Phytantriol (C20H42O3, purity >95\%) was obtained from TCI Chemicals Pvt. Ltd, India and Dodecane (C12H26), was purchased from Otto Chemie Pvt. Ltd., India. Different surfactants, nonionic decaoxyethylene ndodecylether (C12E10)], cationic DTAB (dodecyl trimethylammonium bromide) and anionic SDS (Sodium dodecyl sulfate) were purchased from Sigma-Aldrich. The oil in water $(\mathrm{O} / \mathrm{W})$ microemulsions was prepared using procedure described by Muller [ ]. Samples were prepared by mixing phytantriol and dodecane (DPT) mixture in 50:50 in wt. ratio. The surfactant content kept fixed at $1.0 \mathrm{wt} \%$ while varying the DPT content was varied from 0.1 to $1.0 \mathrm{wt} \%$ in the overall solution to monitor the stabilization efficiency of surfactant against increasing content of DPT. The solution was sonicated by bath sonicator for 1 to 2 hours after addition of surfactants. The samples for SANS measurements were prepared in $\mathrm{D}_{2} \mathrm{O}$ instead of $\mathrm{H}_{2} \mathrm{O}$ to exploit the better scattering length density contrast available for $\mathrm{D}_{2} \mathrm{O}$. DLS measurements were carried out on nanoparticle size analyzer SZ-100 (Horiba, Japan). The wavelength of the incident laser light was $5320 \AA$ and the data were collected at scattering angle $173^{\circ}$. The instrument employs solid state Nd-Yag laser as light source and photomultiplier tube as the detector. Small-angle neutron scattering experiments were performed at SANS-I facility, Swiss Spallation Neutron SourceSINQ, Paul ScherrerInstitut, Switzerland. The necessary corrections were applied for background and empty cell contributions and data were normalized to absolute cross-sectional unit using standard procedures.

\section{DATA ANALYSIS}

In DLSmeasurement, the intensity autocorrelation function $\left[\mathrm{g}^{2}(\tau)\right]$ of scattered light as a function of delay time $(\tau)$ is observed. This correlation function is related to the effective hydrodynamic size $\left(2 \mathrm{R}_{\mathrm{H}}\right)$ of the particles and can be given by [6]

$$
g^{2}(\tau)=A \exp \left(-2 \frac{k T}{6 \pi \eta R_{H}} Q^{2} \tau\right)+B(1)
$$

where A represents a factor related to instrument parameters and B denotes the base line. $\mathrm{k}$ is the Boltzmann's constant, $\eta$ is the solvent viscosity, and $\mathrm{T}$ is the absolute temperature.

In SANS experiments, one measures the coherent scattering intensity as a function of $Q$ which can be given by [7]

$$
I(Q)=n V^{2}\left(\rho_{p}-\rho_{s}\right)^{2} P(Q) S(Q)+B
$$

where $n$ is the number density of particles and $V$ is particle volume. $\rho_{p}$ and $\rho_{\mathrm{s}}$ are scattering length densities of particle and solvent, respectively, wave vector transfer $\mathrm{Q}=(4 \pi \sin \theta) / \lambda ; 2 \theta$ is scattering angle, $\lambda$ is wave length of neutrons. $P(Q)$ is intraparticle structure factor and $S(Q)$ is interparticle structure factor. $B$ is a constant term representing incoherent background. $P(Q)$ accounts for the scattering from a single particle and hence depends on shape and size of the particle whereas $S(Q)$ is Fourier transform of the radial distribution function $\mathrm{g}(\mathrm{r})$ and hence correlates particles present in the system.

\section{RESULTS AND DISCUSSION}

Fig. 1 shows the SANS data of $1 \mathrm{wt} \%$ of different surfactants, namely nonionic decaoxyethylene n-dodecylether (C12E10)], cationic DTAB (tetradecyl dimethyl ammonium bromide) and anionic SDS (Sodium dodecyl sulphate). The SANS data exhibits the monotonic decrease of scattering curve as a function of wave vector $\mathbf{Q}$ for C12E10. At the concentration of $1 \mathrm{wt} \%$, the interaction between the C12E10 can be neglected i.e., $\mathrm{S}(\mathbf{Q}) \sim 1$. However for SDS, the scattering shows small interaction between SDS molecules. In case of DTAB, the scattering profile observed shows well resolved peak indicating the strong interaction among DTAB molecules for $1 \mathrm{wt} \%$ of DTAB. This is due to presence of charge on the surfactant molecules. The structural parameters of the pure components are given in table 1. The data for the mixed systems have been collected at the additive surfactant concentrations after rigorous sonication. To confirm the droplet formation, first DLS measurements were recorded by varying the DPT to C12E10 ratio. Without surfactant ( $0 \mathrm{wt} \% \mathrm{DPT}$ ), The DLS measurement of $1 \mathrm{wt} \% \mathrm{C} 12 \mathrm{E} 10$ displayed a constant auto correlation value of 1 as a function of delay time (not shown in Fig. 2a) implying the continuous oil phase with and without sonication. One of the ways to change the ratio of DPT to surfactant is to fix the DPT wt $\%$ and change 
surfactant $\mathrm{wt} \%$ or fix the surfactant $\mathrm{wt} \%$ and change the DPT. We chose the second method so as to use DPT optimally. The DLS measurement exhibits that on decreasing the DPT $\mathrm{wt} \%$ and consequently increasing the surfactant to DPT ratio, the autocorrelation function gets narrowed (Fig. 2a), indicating the formation of smaller droplet size. To confirm the droplet size and its stability, SANS measurements (Fig. 2b) were carried out after several hours of sample preparation.

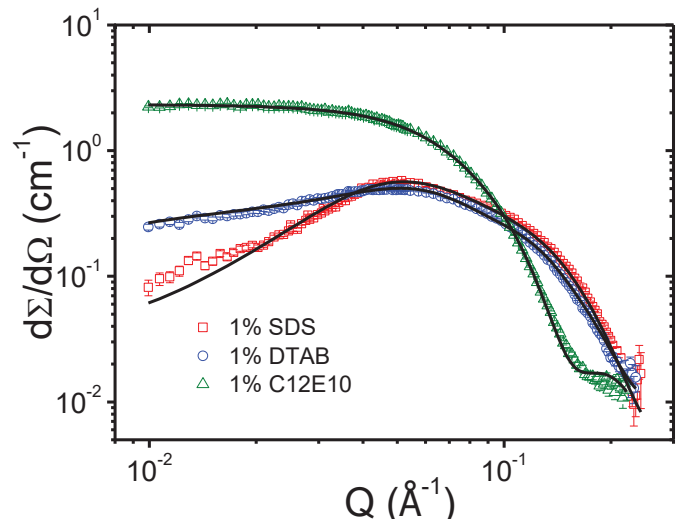

FIGURE 1. SANS data of $1 \mathrm{wt} \%$ of anionic (SDS), cationic (DTAB) and non ionic (C12E10) surfactants.

In SANS, the scattering profile shows an upturn at low Q values suggesting the presence of larger droplet size along with smaller droplets beyond the DPT concentration of $0.5 \mathrm{wt} \%$ and above (smaller C12E10 to DPT ratio). About two length scale.......It isinferred that the significantly higher amounts of additives (C12E10) is required to provoke the formation of stable droplet through steric stabilization mechanism. Nonionic surfactants heads are polymeric chains dissolved in water. These polymeric chains can behave as tiny springs that push off other droplets, stopping them from coming together and hence coalescing. The C12E10 surfactant in sufficient concentration is crucial to create and stabilize the emulsion droplets against coalescence. Another aspect comes out from the measurement is that by varying the concentration of the dispersed phase (DPT) and the surfactant, it is possible to tailor the size of the droplets. The bigger droplets will require larger concentration of C12E10 to stabilize. It can also be noted that the droplets formed (Fig. 2b) do not have any internal structure and no peak corresponding to liquid crystalline phase is observed. The nonionic surfactant C12E10 can stabilize the DPT microemulsions droplets up to 5:1 ratio of surfactant to phytantriol. The destabilization process starts once the C12E10 to DPT ratio is lowered to $2: 1$. In order to investigate
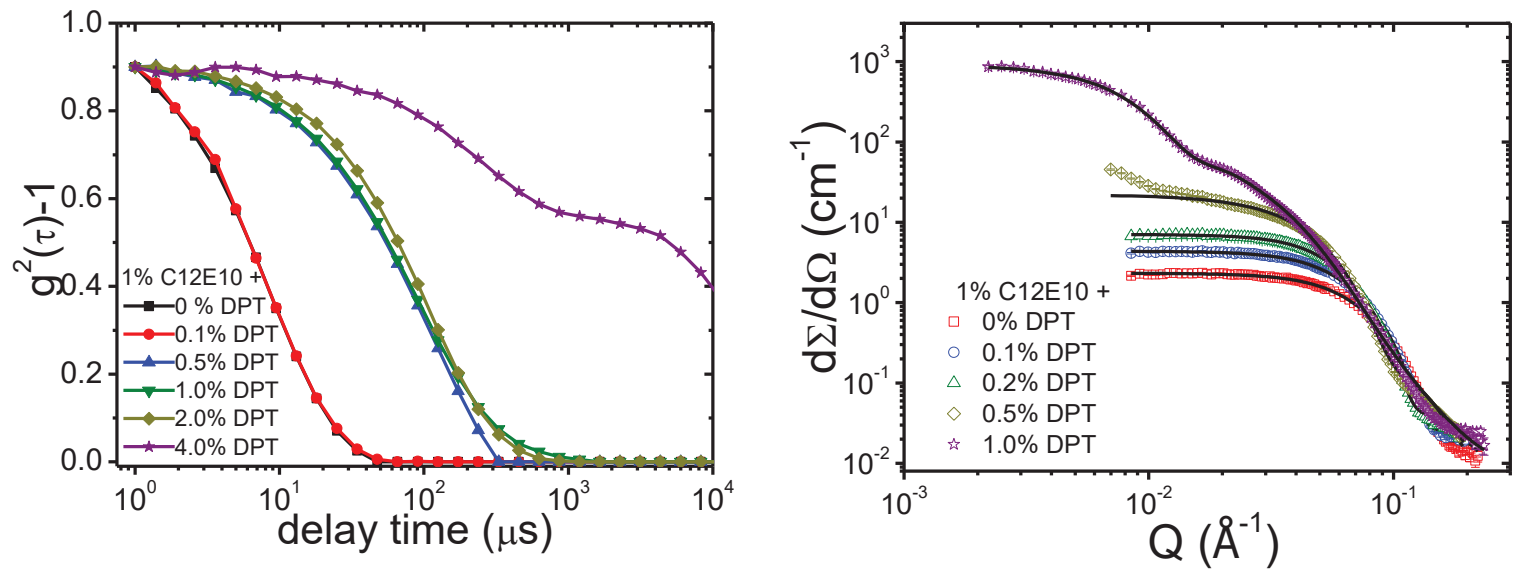

FIGURE 2. DLS (a) and SANS (b) measurements of $1 \mathrm{wt} \%$ non ionic (C12E10) surfactant with varying concentration of dodecane phyatantriol (DPT).

the charge induced stabilization of DPT droplets, microemulsions with anionic SDS and cationic DTAB further examined. Next, we investigated the effect of addition of ionic surfactants in dispersed phase i.e., DPT. DLS and 
SANS measurements were carried out to study the stability and size of the droplet. Surfactants, The way, in which these ionic surfactants adsorb at the oil/water interfaces are governed by the properties of each endof the molecule and dictate the behavior of microemuslion. The hydrophilic heads are attracted to water,while the hydrophobic tailpart aligns with the oil. When the $1 \mathrm{wt} \%$ concentration of an anionic (SDS) surfactant is added to DPT phase, it goes at the O/W interface, making eachdroplets negatively charged. Sincelike charges repel, the SDS helps in preventing the droplets coming together due to electrostatic repulsion. This significantly reduces the droplet coalescence and hence helps in to achieve the stable droplet formation. The SANS data for $1 \mathrm{wt} \%$ SDS and $1 \mathrm{wt} \%$ DPT was recorded. Fig. 3 shows an additional peak at Q $0.11 \AA^{-1}$ (for SDS surfactant) indicating the formation of liquid crystalline phase as observed in bulk phase. This value is in agreement for DPT inverse micelle for O/W microemuslion phase. This peak provides information about an average value of inter miceller distance and also about internal ordering of DPT phase.However, at smaller Q values, there is a strong upturn in scattering curve suggesting the two length scale for the droplets. The droplets can be stabilized by possibly further increasing the surfactant to DPT ratio.

The Cationic surfactant also displays the similar behavior with DPT. The SANS data for $1 \mathrm{wt} \%$ DTAB and $1 \mathrm{wt} \%$ DPT was recorded and shown in Fig. 3. The cationicsurfactant would enable the formation of positively charged droplets and hence will repel the like charged droplets achieving the droplet stability. Like SDS, DTAB also exhibits an additional peak at $\mathrm{Q} \sim 0.11 \AA^{-1}$ indicating the formation of liquid crystalline phase, in agreement with inverse miceller phase in DPT. The scattering curve shows an upturn at smaller Q values, suggesting for the two lengths droplets. However, the smaller upturn for DTAB suggest that smaller surfactant to DPT ratio will be needed to achieve stable and monomodal droplets compared to SDS. It should be noted that ionic surfactant in sufficient concentration is crucial to creating and maintaining theemulsion droplets.

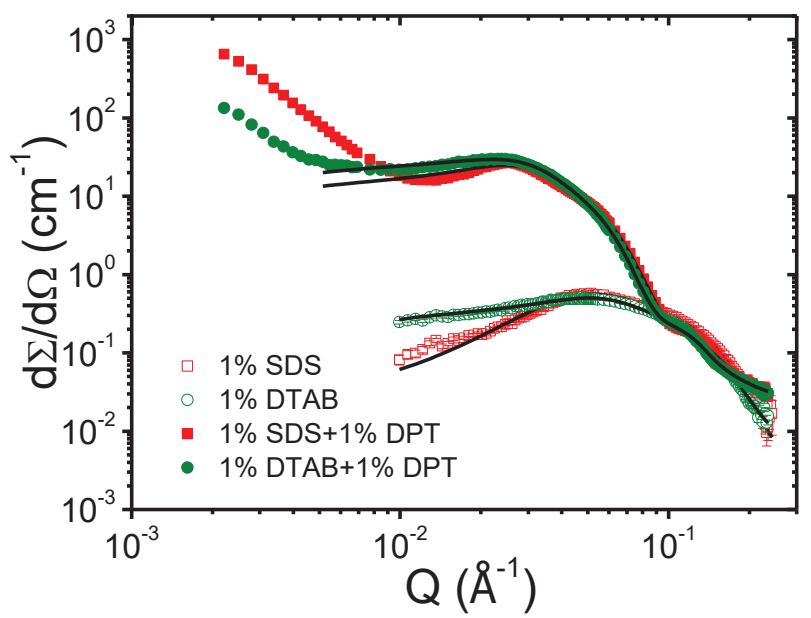

FIGURE 3.SANS measurements of $1 \mathrm{wt} \%$ anionic (SDS), cationic (DTAB) surfactants and with addition of $1 \mathrm{wt} \%$ dodecane phyatantriol (DPT) in each surfactant.

\section{REFERENCES}

1. J. H. Schulman, W. Stoekenius, L.M.Prince, J. Phys. Chem. 63, 1677-1680 (1959).

2. V. Chhabra, , M. L. Free, P. K. Kang, S. E. Truesdail, , D. O. Shah.Tensile Surfact.Det. 34, 156-168 (1997).

3. M. A. Malik, M. Y. Wani, M. A. Hashim, Arabian Journal of Chemistry 5, 397-417 (2012)

4. P. L. Luisi, L. J. Majid, J. H. Fendler,. Crit. Rev. Biochem. 20, 409-474 (1986).

5. F. Muller etal, Soft Matter, 8, 10502-10510 (2012)

6. A. D. Dinsmore, M. F. Hsu, M. G. Nikolaides, M. Marquez,A. R. Bausch and D. A. Weitz, Science, 298, 1006-1009 (2002).

7. J. Gustafsson, H. Ljusberg-Wahren, M. Almgren and K. Larsson,Langmuir, 1996, 12, 4611-4613 (1996).

8. P. A. Hassan, S. Rana, and G. VermaLangmuir31, 3-12 (2015).

9. J. S. Pedersen, Adv. Coll. Inter. Sci.70, 171-210 (1997). 